\title{
LA NO RESPUESTA EN ENCUESTAS PRESENCIALES REALIZADAS EN ESPAÑA
}

\section{NON-RESPONSE IN FACE-TO-FACE SURVEYS IN SPAIN}

\author{
VIDAL DíaZ de RADA vida@unavarra.es \\ Universidad Pública de Navarra. España
}

\begin{abstract}
RESUMEN
El paulatino descenso en la cooperación en las encuestas hace peligrar la viabilidad futura de esta herramienta, una de las mejores para el conocimiento de la sociedad. Desde la última década del pasado siglo numerosas investigaciones han estudiado el fenómeno en profundidad, al tiempo que se ha producido una mayor "transparencia" del proceso de recogida de datos al difundirse documentos donde se detallan con precisión los "sucesos" acontecidos durante el trabajo de campo. En este trabajo se analiza la no respuesta de las encuestas presenciales realizadas en España considerando las que seleccionan los entrevistados con rutas y cuotas, así como las que emplean documentos administrativos como el Padrón de Población. El trabajo termina con una serie de estrategias que han demostrado su eficacia para contrarrestar los efectos de la no respuesta.
\end{abstract}

\section{Palabras clave}

Encuestas presenciales; Mejora de la cooperación; No respuesta total; Representatividad.

\begin{abstract}
The gradual decline of cooperation in surveys jeopardises the viability of this tool in the future, despite it being one of the most useful in gaining knowledge of society. Since the last decade of the 20th century, substantial, in-depth research has been conducted into this social phenomenon, whilst at the same time there has been further "transparency" in the data collection process, as documents are disseminated which describe in detail the "events" that occurred during field work. This paper analyses the lack of response in face-to-face surveys conducted in Spain, considering not only those selecting interviewees by using random route and quotas, but also those using administrative documents, such as the population census. It concludes by providing a number of proven, efficient strategies for counteracting the effects of non-response.
\end{abstract}

\section{KEYWORDS}

Face-to-Face Surveys; Survey Cooperation; Unit Non-Response; Representativeness. 


\section{INTRODUCCIÓN}

La encuesta, una de las herramientas más adecuadas para el conocimiento de la sociedad, está sufriendo actualmente determinados "problemas" que hacen peligrar su viabilidad futura (Stoop et al. 2010). Hace más de medio siglo que se detectó un descenso de la población que responde a encuestas, situación que ha venido aumentando progresivamente $y$, de forma más acusada, desde principios del siglo XXI (Dillman, Smyth y Christian 2009).

Rossi, Wright y Anderson sitúan el inicio del fenómeno de la no respuesta en la década de 1970, aunque Leslie Kish ya expuso —en su clásico libro de muestreo, aparecido en 1965- los problemas originados por la no respuesta y varias estrategias para mitigar su efecto (Kish 1965). De hecho, el análisis de otras fuentes (p.ej. Smith 1981; Steeh 1981) desvela que el descenso se viene produciendo desde 1950, aunque es un fenómeno más acusado a partir de la década de 1970. Aunque desde la década de los sesenta del siglo XX existen investigaciones centradas fundamentalmente en la cooperación en las encuestas por correo (entre otros, Dillman 1978), el número de trabajos sobre el tema de la no respuesta aumentó notablemente en la década de 1980 (entre otros, DeMaio 1980; Steeh 1981; Goyder 1985; Goyder y Leiper 1985; Goyder 1987), si bien no es hasta la última década del siglo XX cuando aparece una línea de investigación específica sobre el tema. Los primeros estudios realizados por Groves, Cialdini y Couper (Groves y Cialdini 1992; Groves, Cialdini y Couper 1992; Groves y Couper 1992 y 1993) son continuados por otros investigadores a ambos lados del Atlántico (entre otros, Bradbrun 1992; Berdie 1993; Goyder 1994; Hox y Deleeuw 1994; Lin y Schaeffer 1995; Willimack et al. 1995; Dillman et al. 1996; Lynn 1996; Campanelli, Sturgis y Purdon, 1997; Campanelli y Skinner 1997; Laurie, Smith y Scott 1997; Sturgis y Campanelli 1997) hasta culminar en el año 1998, en la mejor publicación realizada sobre el tema (Groves y Couper 1998). Para estos autores la cooperación en una encuesta depende de cuatro factores, dos de ellos controlados por el investigador y otros dos que escapan de su control. El entorno social, las características de la persona a entrevistar y la interacción entrevistado-entrevistador escapan del control

Este trabajo es una versión reducida de la ponencia presentada en el Curso de Verano "Las encuestas de opinión en la sociedad actual: desafíos y oportunidades", organizado por la Universidad Complutense de Madrid y el Centro de Investigaciones Sociológicas en julio de 2011. El autor agradece a Ramón Ramos y a Mónica Méndez su invitación al curso, y a dos revisores anónimos su notable ayuda en la mejora del texto. Deseo expresar también mi gratitud al equipo español de la Encuesta Social Europea, en especial a Mariano Torcal y a María José Hierro, al equipo Deusto de Valores Sociales, en especial a Edurne Bartolomé, a J. J. Toharia, de Metroscopia y al Banco de Datos del Centro de Investigaciones Sociológicas. 
del investigador; mientras que las características del entrevistador y del diseño de investigación pueden ser controladas por el mismo investigador.

A partir de este momento se produce un gran desarrollo en esta línea de estudio con la aparición de un gran número de monografías (entre otros, Díaz de Rada 2000; Sánchez Carrión 2000; Groves et al. 2002; Stoop 2005; Eustat 2008; Stoop et al. 2010; Bethlehem, Cobben y Schouten 2011), números monográficos de revistas científicas (entre otras, Journal of Official Statistics 1999 y 2001, Public Opinion Quarterly 2006) y, desde 1990, la celebración anual de un seminario internacional sobre no respuesta (http://www.nonresponse.org) cuya 22. ${ }^{a}$ edición (año 2011) se ha celebrado en España.

La no respuesta genera, en primer lugar, una reducción del tamaño de la muestra, aumentando el error muestral y con ello el error típico de las estimaciones. Se trata de un aspecto escasamente importante puesto que, conocido el problema, aumentar el tamaño muestral en la fase del diseño elimina las reducciones en el tamaño muestral. Más importante es la introducción de sesgos, ya que la no respuesta no es aleatoria, no afecta por igual a todos los estratos de la población, de modo que la muestra obtenida genera sesgos a la hora de estimar el universo total (entre otros Blom, Lynn y Jackle 2008). Así, una baja tasa de respuesta trae como consecuencia que los resultados solo sean representativos de una parte de la población objeto de estudio, no siendo posible la generalización de los mismos, aspecto fundamental en la investigación social mediante encuesta.

Vista la importancia del problema en el ámbito internacional resulta sorprendente el escaso interés sobre el tema en nuestro país, con apenas media docena de publicaciones sobre el tema (De Leeuw y de Heer 2002; Díaz de Rada 2000; Sánchez Carrión 2000; Torcal, Morales y Riva 2006; y Riva, Torcal y Morales 2011), mucho más cuando la tasa de respuesta ha sido empleada en numerosas ocasiones como indicador de "calidad" de la investigación (Biemer y Lyberg 2003). Este trabajo busca contribuir al desarrollo de esta temática analizando la no respuesta de las encuestas presenciales realizadas en España. Para ello centraremos la atención en investigaciones ya realizadas, que, o bien facilitan la información del proceso de recogida o, en su defecto, permiten un fácil acceso a sus datos "brutos". Se trata, en definitiva, de una investigación secundaria (Kiecolt y Nathan 1985; Bulmer, Sturgis y Allum 2009) basada en trabajos realizados por otros colegas.

La exposición comienza con las encuestas presenciales con rutas y cuotas y continúa con las encuestas nominativas basadas en un registro administrativo como el Padrón municipal. Se considera después una encuesta que "actualiza" esta información y, por último, otra que emplea recursos para aumentar la cooperación. El trabajo termina con una serie de estrategias que han demostrado su eficacia para contrarrestar los efectos de la no respuesta. 


\section{Tipología de la no respuesta en los barómetros del Centro de INVESTIGACIONES SOCIOLÓGICAS, REALIZADAS CON RUTAS ALEATORIAS Y CUOTAS}

La mayor parte de la investigación con encuesta presencial realizada en España se lleva a cabo seleccionando las viviendas donde hacer las entrevistas con un sistema de rutas aleatorias, y una segunda selección dentro de la vivienda con el método de cuotas. llustraremos la no respuesta de esta modalidad empleando seis barómetros del Centro de Investigaciones Sociológicas (CIS) correspondientes a los seis primeros meses del año 2011. Centraremos la atención en las incidencias ocurridas del proceso de localización del entrevistado: el número de intentos efectuados para realizar una entrevista 0 , dicho de otra forma, la cantidad de viviendas con las que se ha contactado para llevar a cabo las 2500 encuestas de cada barómetro. En el primer semestre del año 2011 fueron contactadas 333.168 viviendas y se consiguieron 14.827 cuestionarios, lo que supone la realización de 21,47 incidencias por entrevista. Una comparación de los barómetros de 2011 con el número de incidencias detectado en la investigación de Núñez Villuendas en los barómetros realizados entre 1996 y 2003 (88 barómetros y 216.830 entrevistas) desvela un aumento de 10,17 a 21,47, lo que supone duplicar el número de incidencias en quince años.

Tabla 1.

Incidencias en encuestas con rutas aleatorias y cuotas de sexo y edad en los barómetros del Centro de Investigaciones Sociológicas. Porcentajes verticales.

\begin{tabular}{lrr}
\hline & 2004 & 2011 \\
\hline I.2. Dificultad de acceso al edificio, casa, urbanización, etc. & $1,67 \%$ & $2,72 \%$ \\
\hline I.3. Viviendas en las que no hay nadie (ausencias) & $\mathbf{4 8 , 3 3 \%}$ & $\mathbf{5 2 , 4 3 \%}$ \\
\hline I.4. Viviendas en las que se niegan a recibir explicación & $8,18 \%$ & $8,26 \%$ \\
\hline I.5. Negativas de varones a realizar la entrevista & $4,26 \%$ & $3,38 \%$ \\
\hline I.6. Negativas de mujeres a realizar la entrevista & $6,21 \%$ & $5,94 \%$ \\
\hline I.7. Contactos fallidos por no cumplir cuota & $\mathbf{2 6 , 1 8 \%}$ & $\mathbf{2 1 , 5 4 \%}$ \\
\hline I.8. Contactos fallidos por no ser vivienda (oficinas, consultas médicas, etc.) & $3,61 \%$ & $3,01 \%$ \\
\hline I.9. Viviendas de inmigrantes & $1,57 \%$ & $2,73 \%$ \\
\hline Total incidencias & 420.138 & 318.314 \\
\hline Número de entrevistas realizadas & 27.359 & 14.827 \\
\hline Promedio de contactos por cuestionario respondido & 15,36 & 21,47 \\
\hline
\end{tabular}

Fuente: Elaboración propia con datos de los barómetros del CIS 2011. Para el año 2004, Díaz de Rada y Núñez Villuendas 2008. En el año 2011 se han considerado los datos de los seis primeros meses del año 2011. En el anexo se muestra la definición de cada incidencia. 
Conocido el número medio de incidencias - y su evolución- procederemos con un análisis detallado de su distribución en el año 2011. Más de la mitad $(52,43 \%)$ son producidas por llamar a viviendas en las que nadie responde: un $21,54 \%$, por problemas con el cumplimiento de las cuotas; $y$ un $9,32 \%$ por negativas a cooperar, a los que habría que añadir el $8,26 \%$ que cierra la puerta antes de oír que se trata de una encuesta.

El análisis comparativo respecto a la investigación del año 2004 (tabla 1) revela un incremento en las dificultades de acceso a la vivienda, fundamentalmente por las llamadas a viviendas vacías y los problemas de acceso al edificio, y un ligero descenso de los problemas específicos del método de cuotas. Es importante tener en cuenta que estos dos aspectos suponen el $63,41 \%(2,72+52,43+8,26)$ y el $21,46 \%$ de las contactos infructuosos. Incidir sobre ellos producirá, sin duda, mejoras en las tasas de respuesta.

\section{LA NO RESPUESTA DE LAS INVESTIGACIONES BASADAS EN DATOS PADRONALES. El caso del InStituto Nacional de Estadística}

La justificación de analizar aquí las encuestas basadas en registros padronales se fundamenta, en primer lugar, en el gran número de encuestas que realiza el Instituto Nacional de Estadística (INE) y, en segundo lugar, porque se trata de algo habitual en las investigaciones realizadas por Gobiernos autonómicos y otros organismos públicos municipales. Un tercer motivo, no menos importante, es que estos registros son utilizados para importantes investigaciones realizadas desde ámbitos académicos o institucionales, entre los que destacamos la Encuesta Social Europea, las investigaciones del ISSP, o la Encuesta Nacional de Salud (Ministerio de Sanidad y Consumo 2006).

Para conocer la no respuesta en encuestas basadas en el padrón, se han tomado los datos de dos investigaciones recientes, la Encuesta Europea de Salud y la última edición de la Encuesta sobre Equipamiento y Uso de Tecnologías de Información y Comunicación en los hogares. En la Tabla 2 se aprecia que la muestra de la encuesta de salud se reduce de 23.097 unidades a 14.755 viviendas titulares por la presencia de 8.342 incidencias, lo que supone una tasa de respuesta mínima - según la fórmula RR11 de la American Association for Public Opinion Research-AAPOR (2011) - del $63,8 \%$, que obligará a "completar" la muestra con un grupo de reservas.

Comenzando con las incidencias del grupo de titulares, las personas originalmente seleccionadas para formar parte de la muestra, más de un tercio se "pierde" por pro-

\footnotetext{
${ }^{1}$ Se trata de la fórmula más utilizada internacionalmente. Se calcula dividiendo el número de entrevistas realizadas entre el número total de elementos considerados: entrevistas cumplimentadas, entrevistas parciales, rechazos, no contactos, otros incapaces de colaborar, desconocido si el hogar está ocupado, y desconocido otras situaciones.
} 
Tabla 2.

Encuestas basadas en listados padronales: Instituto Nacional de Estadística.

\begin{tabular}{|c|c|c|c|c|}
\hline & \multicolumn{2}{|c|}{$\begin{array}{l}\text { Encuesta Europea de Salud } \\
\qquad 2009\end{array}$} & \multicolumn{2}{|c|}{$\begin{array}{c}\text { Encuesta equipamiento y uso } \\
\text { de tecnologías de información } \\
\text { y comunicación en hogares } \\
2010\end{array}$} \\
\hline \multicolumn{5}{|l|}{ Diseño muestral y empleo de reservas } \\
\hline & $\mathrm{N} .{ }^{\circ}$ casos & $\begin{array}{c}\% \text { respecto a } \\
\text { muestra } \\
\text { teórica }\end{array}$ & N. ${ }^{0}$ casos & $\begin{array}{c}\text { \% respecto a } \\
\text { muestra } \\
\text { teórica }\end{array}$ \\
\hline Muestra teórica $\left({ }^{*}\right)$ & 23.097 & $100,0 \%$ & 6.128 & $100,0 \%$ \\
\hline Encuestables (sin problemas de marco**) & $21.061^{* \star *}$ & $91,2 \%$ & 5.275 & $86,1 \%$ \\
\hline Muestra efectiva (realizada) & 22.188 & $96,1 \%$ & 4.218 & $68,8 \%$ \\
\hline Viviendas titulares & 14.755 & $63,8 \%$ & \multirow{3}{*}{\multicolumn{2}{|c|}{ No contempla reservas }} \\
\hline Viviendas reservas & 7.433 & $32,2 \%$ & & \\
\hline Pérdidas muestrales (no encuestados) & 909 & $3,9 \%$ & & \\
\hline \multicolumn{5}{|c|}{ Distribución porcentual de las incidencias (porcentajes verticales) } \\
\hline & Titulares & Reservas & & \\
\hline Incidencias marco muestral: & $(35 \%)$ & $(31 \%)$ & & $(45 \%)$ \\
\hline \multicolumn{5}{|l|}{ Viviendas no encuestables } \\
\hline Vacía & $28,90 \%$ & $24,24 \%$ & & $36,8 \%$ \\
\hline llocalizable & $3,96 \%$ & $4,39 \%$ & & $4,1 \%$ \\
\hline Destinada a otros fines & $1,99 \%$ & $1,71 \%$ & & $3,2 \%$ \\
\hline Seleccionada anteriormente & $0,22 \%$ & $0,36 \%$ & & \\
\hline Viviendas inaccesibles & $0,38 \%$ & $0,42 \%$ & & $0,5 \%$ \\
\hline Incidencias en hogares & $(65 \%)$ & $(69 \%)$ & & $(55 \%)$ \\
\hline Rechazos (negativas) & $28,46 \%$ & $22,78 \%$ & & $28,3 \%$ \\
\hline Ausencias & $34,56 \%$ & $44,84 \%$ & & $25,6 \%$ \\
\hline Incapacidad para contestar & $1,53 \%$ & $1,27 \%$ & & $1,4 \%$ \\
\hline Total incidencias: & 8.342 & 6.476 & & 1.910 \\
\hline
\end{tabular}

Fuente: Instituto Nacional de Estadística 2009; 2010a. 
blemas con el marco muestral, fundamentalmente por el elevado número de viviendas vacías, un $28,9 \%$. A estas se añade un $3,9 \%$ de ilocalizables. Solventados los errores en el marco muestral, el número de incidencias detectadas dentro de las viviendas casi dobla las anteriores, fundamentalmente por el gran número de ausencias, un 34,5\%. El propio informe del INE señala que "[...] es posible la existencia de trasvases entre viviendas vacías y ausencias (en ambos sentidos), ya que a veces no es posible distinguir entre ambos tipos de incidencia [...]" (Instituto Nacional de Estadística 2009). De hecho, más adelante dice que "[...] algunas de las viviendas consideradas como vacías en Baleares podría tratarse de realmente ausencias" (2009). Para el caso que nos ocupa aquí, lo realmente importante es que la suma de viviendas vacías, ausencias e ilocalizables supone un 67,4\%; cifra similar a la detectada en los barómetros del CIS.

La gran reducción muestral — de 23.097 a 14.755— recomienda la utilización de reservas (Instituto Nacional de Estadística 2009), seleccionados de antemano de igual forma que los titulares. De 13.909 reservas han colaborado 7.433 , de modo que la suma de las 23.097 personas inicialmente contactadas más los reservas implica que se ha intentado entrevistar a 37.006 personas para conseguir 22.188 respuestas, lo que supone una tasa de respuesta "real" del 60\% (según la fórmula RR1).

Los problemas del marco muestral son ligeramente inferiores en el caso de los reservas, fundamentalmente por la disminución del número de viviendas vacías. Lo contrario sucede con las incidencias en los hogares ${ }^{2}$, con un alto número de ausencias que eleva la cantidad de "viviendas sin contacto" (vacías, ausencias e ilocalizables) al $73,5 \%$, cinco puntos por encima de la muestra titular.

La disponibilidad de datos padronales y la utilización de un cuestionario para la evaluación de la falta de respuesta (Instituto Nacional de Estadística 2009) permite conocer la tipología de las incidencias dentro del hogar. Las ausencias son mayores en las viviendas unipersonales, en las habitadas por menores de 40 años y por personas con estudios de Bachiller, Formación Profesional de segundo grado o equivalentes. Los rechazos aumentan en los hogares formados por dos y cuatro miembros, mujeres mayores de 65 años y personas sin estudios (estudios menores que certificado de escolaridad).

La segunda investigación utilizada es la Encuesta sobre Equipamiento y Uso de Tecnologías de la Información y la Comunicación en los hogares del año 2010. Aunque se trata de una encuesta continua, este análisis se centrará en las personas

\footnotetext{
${ }^{2}$ En este texto se emplearán los términos hogar y vivienda indistintamente, en la medida que resulta difícil diferenciarlos con precisión en las unidades muestrales en las que no se establece contacto. Pese a esta forma de proceder, es importante recordar que un "hogar" es un grupo de personas que residen habitualmente en la misma vivienda familiar y comparten los gastos comunes ocasionados por el uso de la vivienda y/o los gastos de alimentación (Díaz de Rada 2005), mientras que la vivienda se refiere al espacio físico donde convive el hogar; de modo que en una vivienda pueden residir varios hogares.
} 
que cooperan por primera vez, un $25 \%^{3}$. Esta investigación consigue una tasa de respuesta ligeramente superior que la Encuesta de Salud, aunque el gran número de viviendas vacías produce un importante aumento de las incidencias del marco. En cualquier caso, la suma de hogares vacíos, ausencias e ilocalizables genera un $66,5 \%$ de viviendas con las que no se ha podido establecer contacto, y los rechazos suponen un $28,3 \%$ de las incidencias.

La comparativa con los registros padronales proporciona una tipología de "no respondientes" muy similar a la mostrada por la Encuesta de Salud. El análisis del Padrón desvela que las ausencias se producen fundamentalmente en los hogares unipersonales, habitados por personas entre 26 y 35 años, con estudios de Bachiller, Formación Profesional de segundo grado o equivalentes, Graduado escolar o equivalente y ocupados. Los rechazos (negativas a cooperar) son mayores en los hogares de dos y tres miembros, con edades de 66 a 74 y 26 a 35 años, y pensionistas.

Emplear dos investigaciones de 2009 y 2010 es adecuado para conocer la situación actual pero, ¿se trata de una situación estable, ¿o más bien la progresiva saturación de encuestas es la responsable de la decreciente participación? La tabla 3 añade a la anterior la comparativa de una investigación similar del 2003, la Encuesta de Empleo del Tiempo (Instituto Nacional de Estadística 2003). La primera parte de la tabla, dedicada al diseño muestral y empleo de reservas, desvela unas cifras similares en cuanto a colaboración de titulares, pero peores cuando se considera el número de reservas o el porcentaje de personas que quedaron sin entrevistar.

El análisis de las incidencias revela unas tasas de viviendas sin contacto, vacías y ausencias similares a las que se obtienen en investigaciones más recientes, y una tasa de rechazos muy superior. Dicho de otro modo, los no contactos se mantienen y las negativas a cooperar han experimentado una disminución desde 2003.

Antes de concluir este apartado, señalaremos un breve apunte común a las investigaciones presentadas aquí, referido a la utilización de reservas para paliar los descensos del tamaño muestral. Estos reservas, elegidos aleatoriamente, ¿serán iguales a los titulares?. El informe de la Encuesta de Salud destaca que se ha sustituido viviendas unipersonales y de más de seis miembros por viviendas de dos, tres y cuatro miembros; así como viviendas con personas con estudios de Formación Profesional de $2 .{ }^{\circ}$ grado (y equivalentes) por personas con titulación inferior al grado de Escolaridad (Instituto Nacional de Estadística 2009). Este hallazgo coincide con el localizado por otras investigaciones, por ejemplo, la Encuesta de Empleo del Tiempo (2003), donde los "hogares sustituidos" muestran un menor nivel de formación (analfabetos, sin estudios, enseñanza de primer grado) y menor tamaño (uno y dos miembros). Los "reservas" tienen un mayor nivel de formación (enseñanza secundaria de primer ciclo y estudios universitarios) y mayor tamaño.

\footnotetext{
${ }^{3}$ No se consideran los que han participado previamente porque la colaboración continua en paneles escapa de los objetivos del trabajo.
} 
Tabla 3.

No respuesta en encuestas realizadas hace más de cinco años:

"Encuesta empleo del tiempo 2003".

\begin{tabular}{|c|c|c|}
\hline \multicolumn{3}{|c|}{ Diseño muestral y empleo de reservas } \\
\hline & N. ${ }^{0}$ casos & $\begin{array}{l}\% \text { respecto a } \\
\text { muestra teórica }\end{array}$ \\
\hline Muestra teórica ${ }^{*}$ ) & 23.880 & $100,0 \%$ \\
\hline Encuestables (sin problemas de marco**) & 21.019 & $88,0 \%$ \\
\hline Muestra efectiva (realizada) & 20.622 & $86,5 \%$ \\
\hline Viviendas titulares & 15.134 & $63,3 \%$ \\
\hline Viviendas reservas & 5.488 & $22,9 \%$ \\
\hline Pérdidas muestrales (no encuestados) & 3.258 & $13,3 \%$ \\
\hline \multicolumn{3}{|c|}{ Incidencias } \\
\hline Incidencias marco muestral & 2.861 & $33,07 \%$ \\
\hline \multicolumn{3}{|l|}{ Incidencias en hogares } \\
\hline Rechazos (negativas) & 2.910 & $33,64 \%$ \\
\hline Ausencias & 2.662 & $30,77 \%$ \\
\hline Incapacidad para contestar & 218 & $2,52 \%$ \\
\hline Total incidencias & 8.851 & $100,0 \%$ \\
\hline $\begin{array}{l}\text { *) Muestra teórica = muestra efectiva (reali } \\
\left.{ }^{* *}\right) \text { Encuestables = muestra teórica }- \text { incide }\end{array}$ & & \\
\hline
\end{tabular}

Fuente: Instituto Nacional de Estadística 2003.

Conclusión similar se obtiene en la Encuesta sobre equipamiento y uso de tecnologías respecto al tamaño del hogar (INE); no obstante, el nivel de estudios muestra la situación opuesta al realizarse una menor sustitución de las personas sin estudios (lo que produce una sobreestimación de las personas con estudios). Estas diferencias entre titulares y reservas, ¿afectarán a las variables de la investigación, esto es, al empleo del tiempo y al equipamiento y uso de tecnologías de información y comunicación? Debe tenerse en cuenta que en la Encuesta de Salud un 32,2\% de las entrevistas se ha realizado a reservas, y un 22,9\% en la Encuesta de Empleo del Tiempo, lo que nos lleva a considerar que, en principio, su bajo tamaño no afectará sustancialmente a los resultados. 


\section{LA NO RESPUESTA DE LAS INVESTIGACIONES BASADAS EN DATOS PADRONALES actualizados. el caso de la Encuesta de Población Activa}

La Encuesta de Población Activa (EPA) es, sin duda, la encuesta más grande y compleja de las que se realizan en nuestro país, y se ha incluido aquí por su estrategia de permanente actualización de las secciones censales. El Instituto Nacional de Estadística tiene entre sus funciones la realización del Censo de Población y Viviendas ${ }^{4}$, función compartida con los organismos oficiales de estadística de las Comunidades Autónomas, que le permiten disponer de listados con los nombres y direcciones de todas las personas que residen en un determinado territorio. La disposición de estos listados facilita enormemente la selección de los entrevistados, siempre que se encuentren actualizados. Utilizando el Censo de Población como marco muestral, la EPA realiza un muestreo bietápico - al igual que las investigaciones vistas anteriormente- sobre 3.500 secciones censales de las 34.600 que componen el país. Ahora bien, teniendo en cuenta que el último Censo se realizó en el año 2001, ¿puede considerarse aceptable utilizar esta información para estudiar un fenómeno en el año 2005? ¿Y qué decir de la selección realizada en los años posteriores, que están aún más lejos de la recogida censal (por ejemplo en los últimos meses del año 2011)?

Para solucionar estos problemas se lleva a cabo una revisión permanente del marco muestral. La EPA realiza una actualización del marco de viviendas para incorporar nuevas viviendas: aquellas que no existían, estaban desocupadas 0 eran destinadas a otras finalidades en el momento de realización del Censo. Se trata de dar probabilidad de selección a los que se han movido, y mantener la equiprobabilidad entre todas las personas que viven en la sección, incluyendo también a los que han cambiado su domicilio y acaban de trasladarse a esa sección.

En la Tabla 4 se presentan los resultados sobre la cooperación en la EPA. Las 58.693 viviendas que componen el marco muestral se reducen a 46.422 - un $82,5 \%$ - debido principalmente al elevado número de viviendas vacías: 11.236. Ahora bien, considerando el número de viviendas sin problemas de marco, la Encuesta de Población Activa consigue entrevistar al $84 \%$ (38.863/46.422) de la muestra, la tasa más alta de todas las investigaciones vistas hasta el momento. Esto se explica por el bajo número de ausencias y rechazos.

El análisis de las incidencias desvela el gran número de viviendas donde no se ha establecido contacto: un $82,5 \%$ están vacías, no han sido localizadas o sus residentes están ausentes. Esta elevada magnitud - superior a la obtenida en las investigaciones vistas anteriormente- resulta sorprendente si se tiene en cuenta que las secciones

${ }^{4}$ Ley 12/1989 de 9 de mayo de la Función Estadística Pública (BOE 11/05/1989). 
Tabla 4.

Encuestas basadas en listados padronales actualizados:

La "Encuesta de Población Activa-EPA" (solo entrevistas en primera visita).

\begin{tabular}{|c|c|c|}
\hline \multicolumn{3}{|c|}{ Diseño muestral } \\
\hline & N. ${ }^{\circ}$ casos & $\begin{array}{l}\% \text { respecto a } \\
\text { muestra teórica }\end{array}$ \\
\hline Muestra teórica $\left({ }^{\star}\right)$ & 58.693 & $100,0 \%$ \\
\hline Encuestables (sin problemas de marco**) & 46.422 & $82,5 \%$ \\
\hline Muestra efectiva (realizada) & 38.863 & $66,2 \%$ \\
\hline \multicolumn{3}{|c|}{ Incidencias } \\
\hline & $\mathrm{N} .{ }^{\circ}$ incidencias & Porcentaje \\
\hline \multicolumn{3}{|l|}{ Incidencias marco muestral: } \\
\hline \multicolumn{3}{|l|}{ Viviendas no encuestables: } \\
\hline Vacía & 11.236 & $56,66 \%$ \\
\hline llocalizable & 541 & $2,73 \%$ \\
\hline Destinada a otros fines & 430 & $2,17 \%$ \\
\hline Viviendas inaccesibles & 64 & $0,33 \%$ \\
\hline \multicolumn{3}{|l|}{ Incidencias en hogares } \\
\hline Rechazos (negativas) & 2.967 & $4,96 \%$ \\
\hline Ausencias & 4.592 & $23,15 \%$ \\
\hline Total incidencias & 19.830 & \\
\hline \multicolumn{3}{|c|}{$\begin{array}{l}\left({ }^{*}\right) \text { Muestra teórica }=\text { muestra efectiva }(\text { realizada })+\text { incidencias } \\
\left({ }^{* *}\right) \text { Encuestables }=\text { muestra teórica }- \text { incidencias marco muestral }\end{array}$} \\
\hline
\end{tabular}

Fuente: Instituto Nacional de Estadística 2010b.

elegidas son permanentemente actualizadas. Aun así, el documento técnico de la investigación señala que este valor puede ser inferior al real. Reproducimos una cita literal de este, donde se abordan ambos temas: la actualización y la posible infraestimación de viviendas no encuestables: "[...] la "actualización" consiste en recorrer la sección a fin de dar de alta a edificios y viviendas de nueva creación, visitar las viviendas vacías y los locales comerciales por si alguno de ellos hubiera pasado a ser vivienda habitada, y verificar los límites de la sección. No se visitan las viviendas habitadas para comprobar si siguen siéndolo, por lo que es posible que el porcentaje de viviendas vacías del marco siga aumentando [...]" (Instituto Nacional de Estadística 2010b:7). Cada 18 meses se realiza un recorrido completo por las secciones incluidas en la muestra. 
El porcentaje de ausencias es muy bajo, el menor de todas las investigaciones realizadas hasta ahora, y se aprecia su tendencia descendente cuando se compara con investigaciones anteriores (Instituto Nacional de Estadística 2010b). Este hecho puede explicarse por el empleo de estrategias para aumentar la colaboración: cartas de presentación personalizadas (dirigidas a una persona concreta), folletos informativos sobre la importancia de colaborar en la encuesta, así como la realización de varias revisitas a los hogares que no responden ${ }^{5}$.

El empleo de estas estrategias ayuda asimismo a explicar el bajo número de rechazos, el menor de todas las investigaciones vistas, y también con una tendencia descendente desde el año 2005 (Instituto Nacional de Estadística 2010b). Un estudio sobre las razones expresadas para rechazar desvela que el $83 \%$ de los rechazos destacan cuatro: un $30 \%$ no expresa ninguna razón; el $22 \%$ reconoce falta de interés; otro $20 \%$ no puede cooperar por enfermedad o por fallecimiento de la persona localizada, y un $11 \%$ habla de exceso de molestia. Otro 7,7\% alude a temor o desconfianza y el 9,3\% restante señala otros motivos. Téngase en cuenta la débil argumentación de las dos principales razones, ningún motivo y falta de interés, y lo fácil que sería convencer al entrevistado para que colaborara. Hablaremos de esto más adelante.

\section{LA NO RESPUESTA DE LAS INVESTIGACIONES BASADAS EN DATOS PADRONALES EMPLEANDO ESTRATEGIAS PARA AUMENTAR LA COLABORACIÓN}

Aunque la EPA utiliza algunos recursos para aumentar la colaboración, es sin duda la Encuesta Social Europea la investigación más completa a la hora de emplear estrategias efectivas para conseguir mayor colaboración. Si la Encuesta de Población Activa es la investigación (con encuesta) más importante realizada en nuestro país - en cuanto a su volumen (tamaño muestral, coste e implicaciones de los resultados; según Losilla 2005) - la Encuesta Social Europea (ESE) es, sin ninguna duda, la mejor encuesta de todas las que se realizan actualmente. Tendremos tiempo para demostrarlo.

La ESE utiliza una selección bietápica en la que se eligen municipios y secciones. Dentro de cada sección utiliza un listado de personas proporcionado por el INE y realiza -en la cuarta ola - hasta siete visitas en las viviendas donde no se establece contacto. El tamaño de la muestra teórica de la edición del año 2008, 3.962 unidades, se reduce a 3.203 por problemas con el marco muestral. Eliminadas las viviendas con problemas de marco, esto es, considerando únicamente el número de viviendas encuestables, la tasa de cooperación (2.576/3.338) alcanza el 77\%; es decir, tres de cada cuatro contactados cooperan en la investigación.

\footnotetext{
${ }^{5}$ Es preciso señalar el limitado uso de esta última estrategia porque -al estar el cuestionario referido a una semana concreta - retrasar la entrevista puede generar errores de medida por olvidos del entrevistado.
} 
En la tabla 5 puede observarse que el $61,3 \%$ de las incidencias están generadas por falta de contacto con las viviendas, principalmente por el elevado número de viviendas vacías. Es importante precisar que el marco muestral de esta encuesta lo elabora el INE utilizando el padrón continuo; es el mismo marco que las investigaciones vistas con anterioridad. Sorprende el alto número de rechazos, cercano al $40 \%$, aunque es un porcentaje que se ha reducido notablemente desde el año 2002. Las principales razones de rechazo en la edición del año 2008 están relacionadas con la falta de tiempo/ muy ocupado (93\%), dificultad del tema (29\%), inoportunidad de la visita/mal momento $(21 \%)$, e invasión de la intimidad (16\%), según información proporcionada por el equipo español de la ESS (2009).

La "fragilidad" de las razones de los entrevistados para no participar generó, a principios de la década de 1980, el desarrollo de una estrategia de conversión de rechazos

Tabla 5.

Listados padronales y empleo de estrategias para mejorar la colaboración: "Encuesta Social Europea" (cuarta ola, año 2008).

\begin{tabular}{|c|c|c|}
\hline \multicolumn{3}{|c|}{ Diseño muestral } \\
\hline & N. ${ }^{0}$ casos & $\begin{array}{l}\% \text { respecto a muestra } \\
\text { teórica }\end{array}$ \\
\hline Muestra teórica $\left({ }^{*}\right)$ & 3.962 & $100,0 \%$ \\
\hline Encuestables (sin problemas de marco*) & 3.338 & $84,2 \%$ \\
\hline Muestra efectiva (realizada) & 2.576 & $65,0 \%$ \\
\hline \multicolumn{3}{|c|}{ Incidencias } \\
\hline & N. ${ }^{\circ}$ incidencias & Porcentaje \\
\hline \multicolumn{3}{|l|}{ Incidencias marco muestral: } \\
\hline \multicolumn{3}{|l|}{ Viviendas no encuestables } \\
\hline Vacía (no disponibles, no contactos) & 510 & $36,9 \%$ \\
\hline llocalizable (ilocalizables reales, difícil de encontrar) & 105 & $7,6 \%$ \\
\hline Otros (citas pendientes) & 9 & $0,6 \%$ \\
\hline \multicolumn{3}{|l|}{ Incidencias en hogares } \\
\hline Rechazos (negativas) & 527 & $38,1 \%$ \\
\hline Ausencias & 232 & $16,8 \%$ \\
\hline Total incidencias & 1.383 & \\
\hline \multicolumn{3}{|c|}{$\begin{array}{l}\left({ }^{\star}\right) \text { Muestra teórica }=\text { muestra efectiva }(\text { realizada })+\text { incidencias } \\
\left({ }^{* *}\right) \text { Encuestables = muestra teórica }- \text { incidencias marco muestral }\end{array}$} \\
\hline
\end{tabular}

Fuente: Metroscopia 2009. 
basada en el planteamiento que un "no" puede ser un "no ahora" más que un "no, nunca" (Morton Williams 1983; Campanelli 1997; National Centre for Social Research 1999). Estos autores proponen establecer un segundo encuentro con la vivienda, bien por el mismo entrevistador o por otro con mayor experiencia, y siempre considerando la reacción del entrevistado que manifestó una negativa, esto es, el "tipo de negativa" expresado. La conversión de rechazo no se emplea cuando alguien expresa claramente que no desea colaborar en la encuesta.

Considerando las razones de no cooperación con la ESE señaladas dos párrafos más atrás, con las personas que hablan de "mal momento" se busca fijar una cita posterior; los que señalan que "la encuesta es muy difícil" podrían cambiar de opinión si el encuestador explicara que le interesa su opinión, no sus conocimientos, a los que "no les interesa" le plantea el interés de participar en una investigación realizada conjuntamente en toda Europa, etc. Se trata, en definitiva, de utilizar un argumento que contrarreste el expresado por el entrevistado con el fin de cambiar su actitud (Groves, Singer y Corning 2000).

En la tabla 6 se presentan los resultados conseguidos tras emplear la conversión de rechazos. En el año 2006 únicamente se trató de convertir 212 negativas, de las

Tabla 6.

Conversión de rechazos en la "Encuesta Social Europea".

\begin{tabular}{|c|c|c|c|c|c|c|}
\hline \multicolumn{7}{|c|}{$\begin{array}{l}\text { Año } 2006 \text { (tercera ola) } \\
\text { Número de casos que reciben al menos un rechazo: } 770\end{array}$} \\
\hline \multicolumn{7}{|c|}{ Situación final } \\
\hline & & \multicolumn{2}{|c|}{ No convertidos } & \multicolumn{2}{|c|}{ Convertidos } & N. ${ }^{\circ}$ casos \\
\hline \multirow{2}{*}{ Decisión de convertir el rechazo } & sí & 58 & $27,4 \%$ & 154 & $72,6 \%$ & 212 \\
\hline & NO & 558 & $100,0 \%$ & 0 & $0,0 \%$ & 558 \\
\hline \multicolumn{7}{|c|}{ Convertidos / total rechazos: 154 / $770=20 \%$} \\
\hline \multicolumn{7}{|c|}{$\begin{array}{l}\text { Año } 2008 \text { (cuarta ola) } \\
\text { Número de casos que reciben al menos un rec }\end{array}$} \\
\hline \multicolumn{7}{|c|}{ Situación final } \\
\hline & & \multicolumn{2}{|c|}{ No convertidos } & \multicolumn{2}{|c|}{ Convertidos } & N. ${ }^{\circ}$ casos \\
\hline \multirow{2}{*}{ Decisión de convertir el rechazo } & sí & 111 & $36,0 \%$ & 197 & $63,96 \%$ & 308 \\
\hline & NO & 505 & $100,0 \%$ & 0 & $0,0 \%$ & 505 \\
\hline \multicolumn{7}{|c|}{ Convertidos / total rechazos: $197 / 813=24 \%$} \\
\hline
\end{tabular}

Fuente: Reproducido de Metroscopia 2007 y Metroscopia 2009. 
cuales se obtuvo colaboración en un $72,6 \%$. Estos 154 casos recuperados suponen un $20 \%$ del total de rechazos. En la cuarta ola (año 2008) el número de rechazos aumentó hasta los 813 , de los cuales se trataron de convertir 308. La efectividad en la conversión alcanzó el 64\%, consiguiendo recuperar 197 entrevistados que suponen, en este caso, un $24 \%$ de la muestra.

Además de las siete revisitas y la conversión de rechazos, esta encuesta emplea otras estrategias para aumentar la colaboración (Riba, Torcal y Morales 2010): los seleccionados reciben dos cartas de presentación previas a la visita del entrevistador (tres cuando rechaza cooperar), una gratificación de doce euros, y un folleto con los resultados de las olas previas. Se lleva también a cabo una cuidadosa formación y control de los encuestadores, retribuidos con un salario un $30 \%$ superior al promedio, salario que se incrementa según el número de encuestas y la tasa de respuesta lograda: 10\% extra por entrevista en cada grupo de 24 encuestas, extra del 15-20\% para quien realice más del $70 \%$ de las asignadas (50), y otro extra de un $15-20 \%$ para quienes realicen más del $90 \%$ de las asignadas (Riba, Torcal y Morales 2010).

Las primeras estrategias (revisita, conversión de rechazos, cartas de presentación, folleto de resultados y gratificación) se utilizan para "modificar" la actitud del seleccionado, y el resto para mejorar el rendimiento del equipo de encuestadores. Todo esto genera, lógicamente, tasas elevadas de cooperación, que llegan hasta el 65\% en la cuarta ola. Salvo la EPA, ninguna de las encuestas realizadas en nuestro país logra resultados similares, pese a que las encuestas del INE incluidas en el Plan Estadístico Nacional son de obligado cumplimiento. Aunque la negativa a responder puede ser sancionada, hasta ahora la sanción sólo se ha aplicado a empresas y nunca se ha sancionado a un hogar ${ }^{6}$.

\section{ESTRATEGIAS PARA REDUCIR LA NO RESPUESTA}

Reducir el efecto de la no respuesta requiere, por un lado, del empleo de estrategias que aumenten la cooperación (prevención) y, por otro, llevar a cabo determinadas modificaciones en la información recogida mediante imputación y ponderación (entre otros Blom, Lynn y Jäckle 2008; Matsuo et al. 2011). Consideraremos las primeras, porque son las que efectivamente producen reducciones de la no respuesta, buscando una mejora en el acceso a la vivienda (para diferenciar con precisión las viviendas no encuestables de las ausencias/no localizados) y una reducción del número de negativas o rechazos a cooperar (Schmeets 2010).

\footnotetext{
${ }^{6}$ Información proporcionada por la Subdirección General de Muestreo y Recogida de Datos del Instituto Nacional de Estadística, a los que deseamos expresar nuestro agradecimiento.
} 
Considerando que la mayor parte de la no respuesta en encuestas presenciales se produce por el "no contacto", la primera estrategia busca mejorar el acceso a la muestra objetivo con el fin de diferenciar con precisión las viviendas no encuestables de las "ausencias". La mejor forma de lograr este propósito es aumentando el número de contactos a cada unidad muestral en diferentes momentos del día. Realizar varias visitas a un hogar reduce significativamente el número de ausencias, al tiempo que permite delimitar con más precisión si la vivienda forma parte del universo objeto de estudio (permite conocer si se trata de una vivienda habitada, un negocio, una vivienda vacía, etc.). De hecho, el número de entrevistas realizadas en el primer contacto es muy bajo: un $6,4 \%$ en los barómetros del CIS (primer semestre del 2011) y un 18,7\% en la tercera ola de la ESS, muy inferior a la efectividad de otros países que consiguen hasta un $50 \%$ (Groves et al. 2001). Con cuatro visitas al hogar, la tercera ola de la ESS entrevistó al $75 \%$ de la muestra, y demostró que la efectividad se reduce notablemente a partir del quinto contacto (Riba, Torcal y Morales 2010). De hecho, después de siete visitas no se contactó con un $20 \%$ de la muestra, quedando definidos como no contactos o inelegibles (Riba, Torcal y Morales 2010).

La probabilidad de éxito en el contacto está muy relacionada con el momento del día y con el día de la semana en que se efectúa. En los barómetros, un $66 \%$ de los encuestados sin incidencias (primera visita) fueron entrevistados entre las 9 y las 16 horas, porcentaje que se reduce en cinco puntos en los entrevistados en las visitas posteriores. En la segunda ola de la ESS, el $73 \%$ de los contactos realizados en el mediodía del fin de semana consiguieron una entrevista, porcentaje que se redujo al $69 \%$ en los realizados durante todo el fin de semana; y al $67 \%$ los días laborables entre las 13 y las 16 horas (Torcal, Morales y Riva 2006). Estas tasas de éxito explican que la tercera ola de la Encuesta Social Europea en España exigiera un mínimo de siete intentos a las unidades no contactadas, de las cuales, al menos dos debían ser realizadas en horario vespertino y otras dos en fin de semana (Riba, Torcal y Morales 2010). Contar con los nombres y direcciones de las personas a entrevistar ha facilitado notablemente el trabajo.

Otra de las ventajas de esta estrategia es que amplía la variabilidad muestral al localizar -e incluir en la muestra - las personas que menos tiempo pasan en casa, colectivos con rasgos sociodemográficos específicos (Peytchev, Baxter y Carley-Baxter 2009; Stoop et al. 2010). En el primer contacto son entrevistadas un gran número de mujeres, mayores de 55 años, casados, personas con bajos estudios, retirados y amas de casa, tal y como puede apreciarse en la primera columna de la Tabla 7 . A partir del tercer contacto aumenta el número de hombres, jóvenes y edades medias, solteros, estudios medios, trabajadores (el $61 \%$ de los entrevistados en el cuarto contacto trabajan, aumentando hasta el $65 \%$ en el siguiente) y con niños en el hogar. Todo ello explica que se trate de la estrategia más utilizada a nivel internacional, según la investigación realizada por Smith (2007) en 29 países (utilizada por el $90 \%$ de los investigadores consultados).

La segunda estrategia para reducir la no respuesta se centra específicamente en las negativas a cooperar, negativas que pueden ser mitigadas de tres formas: 
- Empleando estrategias de conversión de negativas. Así, por ejemplo, la Encuesta Social Europea en España utiliza entrevistadores más experimentados para realizar la conversión de los rechazos "suaves" bajo la premisa de que estos serán más persuasivos que los primeros (Riba, Torcal y Morales 2010). En la Tabla 6 dimos cuenta de la efectividad de esta estrategia, $72,6 \%$ de convertidos en la tercera ola y $64 \%$ en la cuarta, muy superiores a las cifras conseguidas en otros países (entre el $11 \%$ en Chequia y el $47 \%$ en los Países Bajos según Stoop et al. 2010) y a las logradas por otras encuestas en países de nuestro entorno como el Reino Unido, donde oscilan entre un 10 y un $20 \%$ (Lynn et al. 2002).

Tabla 7.

Cambios en la respuesta según el contacto en el que se consigue la entrevista en la "Encuesta Social Europea" (porcentajes verticales).

\begin{tabular}{|c|c|c|c|c|c|}
\hline & \multicolumn{5}{|c|}{ Número de contacto } \\
\hline & Primero & Segundo & Tercero & Quinto & Total \\
\hline \multicolumn{6}{|l|}{ Sexo } \\
\hline Hombre & $41 \%$ & $47 \%$ & $53 \%$ & $52 \%$ & $47 \%$ \\
\hline Mujer & $60 \%$ & $53 \%$ & $47 \%$ & $48 \%$ & $53 \%$ \\
\hline \multicolumn{6}{|l|}{ Edad } \\
\hline De 18 a 24 años & $7 \%$ & $10 \%$ & $11 \%$ & $10 \%$ & $10 \%$ \\
\hline De 25 a 34 años & $12 \%$ & $20 \%$ & $23 \%$ & $20 \%$ & $19 \%$ \\
\hline De 35 a 44 años & $17 \%$ & $19 \%$ & $21 \%$ & $21 \%$ & $20 \%$ \\
\hline De 45 a 54 años & $14 \%$ & $15 \%$ & $15 \%$ & $19 \%$ & $16 \%$ \\
\hline De 55 a 64 años & $17 \%$ & $11 \%$ & $11 \%$ & $15 \%$ & $14 \%$ \\
\hline 65 y más años & $32 \%$ & $25 \%$ & $18 \%$ & $14 \%$ & $22 \%$ \\
\hline \multicolumn{6}{|l|}{ Estado civil } \\
\hline Casado & $59 \%$ & $54 \%$ & $50 \%$ & $52 \%$ & $55 \%$ \\
\hline Convive pareja & $2 \%$ & $3 \%$ & $5 \%$ & $3 \%$ & $3 \%$ \\
\hline Separado, divorciado & $5 \%$ & $5 \%$ & $7 \%$ & $7 \%$ & $6 \%$ \\
\hline Viudo & $10 \%$ & $9 \%$ & $7 \%$ & $9 \%$ & $8 \%$ \\
\hline Nunca casado & $23 \%$ & $30 \%$ & $31 \%$ & $30 \%$ & $28 \%$ \\
\hline \multicolumn{6}{|l|}{ Presencia de niños en el hogar } \\
\hline Sí & $41 \%$ & $44 \%$ & $40 \%$ & $42 \%$ & $42 \%$ \\
\hline No & $59 \%$ & $56 \%$ & $60 \%$ & $68 \%$ & $58 \%$ \\
\hline Tamaño del hogar (n. ${ }^{\circ}$ miembros) & 2,94 & 3,1 & 2,4 & 2,98 & 2,99 \\
\hline Total (número de casos) & 652 & 735 & 476 & 187 & 2.576 \\
\hline
\end{tabular}

Fuente: Elaboración propia con datos cedidos por el equipo español de la Encuesta Social Europea (cuarta ola, año 2008). 
- Volver a contactar con el entrevistado, bien realizando un submuestreo entre los rechazos o bien aplicándoles un breve cuestionario para identificar sus rasgos (y así aumentar el peso específico de las personas peor representadas).

- Una tercera posibilidad es reemplazar a los que no cooperan, empleando reservas que sustituyan las ausencias y los rechazos. Se trata de la estrategia muy utilizada en las encuestas que seleccionan a los entrevistados con rutas y cuotas, aunque presenta muchos problemas como señalan, entre otros, Díaz de Rada (2000) y Sánchez Carrión (2000).

Otra forma de reducir el rechazo es entregando gratificaciones a los encuestados, tal y como procede la Encuesta Social Europea cuando entrega un cheque-regalo de doce euros al inicio del primer contacto. Las gratificaciones monetarias producen aumentos de respuesta mayores que las no monetarias, y las entregadas antes de responder el cuestionario son más efectivas que las entregadas después (Van den Brakel, VisVisschers y Schmeets 2006).

\section{Conclusiones}

A mediados de 1970 se detecta por primera vez una menor cooperación de la sociedad norteamericana en las encuestas (Rossi, Wright y Anderson 1983:9), situación que es atribuible -en un principio- al aumento de la criminalidad en las ciudades y al menor número de personas que están en sus viviendas durante el día. Años más tarde, Smith (1995:168) añade nuevas explicaciones al fenómeno relacionadas fundamentalmente con la dificultad en el contacto. El incremento del número de mujeres que trabajan a tiempo completo fuera del hogar y la reducción del tamaño de los hogares plantea dificultades en el contacto porque el hogar está vacío la mayor parte del día. Esta es, de hecho, la situación que explica la no respuesta en las encuestas presenciales realizadas en nuestro país. Como se ha señalado a lo largo del trabajo la encuesta presencial se caracteriza por un bajo número de rechazos, entre el $10 \%$ y el $25 \%{ }^{7}$, y un elevado número de no contactos -entre el $63 \%$ de los barómetros del CIS y el $82 \%$ de la EPA-, si bien la mayoría de las encuestas analizadas se sitúan más cerca del primero. En la Tabla 8 se muestra una síntesis de las diferencias entre la muestra proyectada y la realizada, el número total de contactos realizados y la tasa de respuesta mínima utilizando la fórmula RR1 de la AAPOR: entrevistados entre unidades consideradas, sean contactadas o $\mathrm{no}^{8}$.

\footnotetext{
${ }^{7}$ No olvidamos que la Encuesta Social Europea consigue una tasa de rechazo mayor, pero esto está provocado por el elevado número de contactos que reciben las viviendas no localizadas.

${ }^{8}$ No son pocos los expertos que se muestran muy críticos con esta formulación (Skaland 2011).
} 
Tabla 8.

Tasa de respuesta en varias investigaciones realizadas en España con encuestas presenciales.

\begin{tabular}{|c|c|c|c|}
\hline Muestra teórica (proyectada) & $\begin{array}{l}\text { Muestra } \\
\text { real }\end{array}$ & $\begin{array}{l}\text { Personas contactadas } \\
\text { Tasa respuesta mínima } \\
\text { (RR1) }\end{array}$ & $\begin{array}{l}\text { Problemas de marco } \\
\text { muestral }\end{array}$ \\
\hline \multicolumn{4}{|l|}{ Rutas y cuotas } \\
\hline Barómetros CIS 2004 & 27.500 & 27.359 & 420.138 \\
\hline Barómetros CIS 2011 & 15.000 & 14.827 & 318.314 \\
\hline \multicolumn{4}{|l|}{ Registros padronales } \\
\hline Enc Europea de Salud 2009 & 23.097 & 22.188 & \\
\hline Enc tecnologías en los hogares & 6.128 & 4.218 & 6.128 \\
\hline Enc empleo tiempo 2003 & 23.880 & 20.622 & $\begin{array}{l}\text { Se desconoce el número } \\
\text { de reservas empleados }\end{array}$ \\
\hline \multicolumn{4}{|l|}{ Registros padronales actualizados } \\
\hline Enc Población Activa 2010 & 58.693 & 38.863 & 58.693 \\
\hline \multicolumn{4}{|c|}{ Registros padronales con estrategias de cooperación } \\
\hline Encuesta Social Europea 2008 & 3.962 & 2.576 & 3.962 \\
\hline \multicolumn{4}{|c|}{$\begin{array}{l}\text { Notas: No se calcula la tasa de respuesta en los barómetros porque el método de cuotas no es un método } \\
\text { estrictamente probabilístico. } \\
\text { Los problemas en el marco muestral en los barómetros corresponden a los contactos realizados con "no vivien- } \\
\text { das" (incidencia I.8) y con viviendas de inmigrantes (incidencia I.9). } \\
\text { En la EPA y en la Encuesta de Tecnologías de la Información en los hogares se han considerado únicamente las } \\
\text { entrevistas en primera visita, no las entrevistas sucesivas a los hogares ya seleccionados. } \\
\text { La "tasa de respuesta mínima" se calcula dividiendo la muestra real entre el número de personas contactadas. } \\
\text { La "tasa de respuesta RR5" no considera las viviendas que no pertenecen al marco muestral: vacías, ilocalizadas, } \\
\text { otros fines e inaccesibles. }\end{array}$} \\
\hline
\end{tabular}

Fuente: Tablas anteriores.

La exposición realizada hasta ahora hace dudar de la adecuación de esta fórmula en España, el país en el que más viviendas se han construido en los últimos años, y que cuenta con un gran parque de viviendas vacías. El Censo del año 2001 las estimaba en 3,10 millones, que suponían un 15\% respecto al total, pero es probable que la "fiebre constructora de la primera década del milenio" haya incrementado esta cifra notablemente ${ }^{9}$. Si las viviendas no son parte del universo objeto de estudio, ¿por qué deben formar parte de la formulación de la tasa de respuesta? Mucho más

${ }^{9}$ De hecho, los tres últimos informes de campo de la Encuesta Social Europea hablan de un 15-18\% de viviendas difíciles de encontrar y/o realmente inelegibles (Demoscopia 2006; Demoscopia 2007 y Demoscopia 2011). 
cuando investigaciones como la EPA y la ESE, que realizan un minucioso estudio del marco muestral (bien con la actualización o con el empleo de revisitas), las localizan permanentemente como "problemas del marco". En relación con esto, en la última columna de la citada tabla se muestra el aumento en la tasa de respuesta cuando se eliminan las incidencias relacionadas con el marco muestral (fórmula RR5), esto es, cuando se consideran únicamente las unidades contactadas, bien sean entrevistados, rechazos, ausencias 0 incapaces de responder ${ }^{10}$. Esto permite acercarnos a los consejos de Singleton y Stratis (2005) cuando señalan que tasas de respuesta inferiores al $70 \%$ tienen un serio riesgo de producir estimaciones sesgadas. Bien es verdad, por otro lado, que un meta-análisis realizado por Groves y Peytcheva en 2008 localiza tasas de respuesta entre el 14 y el $72 \%$, con una tasa de respuesta media del $36 \%$.

Para terminar, señalamos que el estudio de la no respuesta ha generado, además, una mayor "transparencia" en la recogida de datos, en la medida en que numerosos organismos (Instituto de Estadística de Euskadi-EUSTAT, Institute for Social and Economic Research de la Universidad de Essex, Instituto de Estudios Sociales Avanzados, Instituto Nacional de Estadística, Statistics Canada, Statistics Norway, Statistics Netherlands, U.S. Census Bureau, etc.) elaboran publicaciones sistemáticas donde se detallan con precisión todos los "sucesos" acontecidos durante el período de recogida de información. Del mismo modo, otros organismos (entre otros, el Centro de Investigaciones Sociológicas-CIS, European Social Survey-ESS, Word Values SurveyWVS o el International Social Survey Programme-ISSP) "colocan" sus datos brutos -con todas las incidencias - en lugares accesibles para que sean utilizados por otros investigadores (Stoop et al. 2010).

\section{REFERENCIAS BibLIOGRÁFICAS}

American Association for Public Opinion Research-AAPOR. 2011. Standard Definitions: Final Dispositions of Case Codes and Outcome Rates for Surveys. AAPOR. Consulta el 13 de junio de 2011 (http:// www.aapor.org/AM/Template.cfm?Section=Standard_Definitions\&Template=/CM/ContentDisplay. cfm\&ContentID=1819).

Benthlehem, J., F. Cobben y B. Schouten. 2011. Handbook of Nonresponse in Household Surveys. Nueva York: Wiley.

Berdie, D. R. 1993. "Telephone Survey Response Rates: How high is high enough?." Marketing Research 5:35-44.

Biemer, P. y L. Lyberg. 2003. Introduction to Survey Quality. Nueva York: Wiley.

${ }^{10}$ De forma similar opera la última investigación sobre la no respuesta en la ESE (Stoop et al. 2010). 
Blom, A., P. Lynn y A. Jäckle. 2008. "Understanding cross-national differences in unit non-response: the role of contact data." ISER Working Papers Series 2008-01, noviembre. Colchester: University of Essex. Consultado 11 de junio de 2011 (http://www.iser.essex.ac.uk/publications/working-papers/iser/2008-01).

Bulmer, M., P. J. Sturgis y N. Allum 2009. The Secondary Analysis of Survey Data. Thousand Oaks, CA: Sage.

Bradburn, N. M. 1992. "A Response to the Nonresponse Problem." Public Opinion Quarterly 56:391-397.

Campanelli, P., P. Sturgis y S. Purdon. 1997. Can you hear me knocking: An investigation into the impact of interviewers on surveys response rates. Londres: SCPR.

Campanelli, P. y C. Skinner 1997. "Curso sobre 'Handling Non-Response'." Curso impartido por el CASS, Universidad de Southampton. 10-12 de marzo. Southampton.

Centro de Investigaciones Sociológicas. 2011. Barómetro de enero. Estudio 2.859. Madrid: CIS.

Centro de Investigaciones Sociológicas. 2011. Barómetro de febrero. Estudio 2.861. Madrid: CIS.

Centro de Investigaciones Sociológicas. 2011. Barómetro de marzo. Estudio 2.864. Madrid: CIS.

Centro de Investigaciones Sociológicas. 2011. Barómetro de abril. Estudio 2.865. Madrid: CIS.

Centro de Investigaciones Sociológicas. 2011. Barómetro de mayo. Estudio 2.888. Madrid: CIS.

Centro de Investigaciones Sociológicas. 2010. Barómetro de junio. Estudio 2.905. Madrid: CIS.

De Leeuw, E. y W. de Heer. 2002. "Trends in household survey nonresponse: a longitudinal and international comparison." Pp. 41-54 en Survey Nonresponse, editado por Robert M. Groves et al. Nueva York: Wiley.

DeMaio, T. 1980. "Refusals: Who, where and why." Public Opinion Quarterly 44:222-233.

Díaz de Rada, V. 2000. Problemas originados por la no respuesta en investigación social: Definición, control y tratamiento. Pamplona: Universidad Pública de Navarra.

Díaz de Rada, V. 2005. Manual del trabajo de campo en la encuesta. Madrid: CIS, Cuadernos Metodológicos 36.

Díaz de Rada, V. 2010. Comparación entre los resultados proporcionados por encuestas telefónicas y personales: el caso de un estudio electoral. Madrid: CIS.

Dillman, Don A. 1978. Mail and Telephone Surveys. Nueva York: Willey.

Dillman, Don A., E. Singer, J. R. Clark y J. B. Treat. 1996. "Effects of Benefits Appeals, Mandatory Appeals, and Variations in Statements of Confidentiality on Completion Rates for Census Questionnaires." Public Opinion Quarterly 60:376-389.

Dillman, D. A., J. D. Smyth y L. M. Christian. 2009. Internet, Mail and Mixed-Mode Surveys: The Tailored Design Method. Nueva York: Wiley.

Eustat. 2008. Estudio y ajuste de la no respuesta en las encuestas a hogares. Vitoria: Eustat.

Goyder, J. 1985. "Face to face interviews and mailed questionnaires: The Net difference in response rate." Public Opinion Quarterly 49:234-252.

Goyder, J. 1987. The Silent Minority. Cambridge: Polity Press.

Goyder, J. 1994. "An experiment with cash incentives on a personal interview survey." Journal of the Market Research Society 36:361-366. 
Goyder, J. y J. McKenzie Leiper 1985. "The decline in survey response: A social values interpretation." Sociology 19:55-71.

Groves, R. M. y R. B. Cialdini 1992. "Toward a Useful Theory of Survey Participation." Pp. 105-120 en Proceedings of the Section on Survey Research Methods. American Statistical Association.

Groves, R. M., Robert B. Cialdini y Mick P. Couper. 1992. "Understanding the decision to Participate in a Survey." Public Opinion Quarterly 56:475-495.

Groves, R. M. y Mick P. Couper 1992. "Correlates of Nonresponse in Personal Visit Surveys." Pp. 102-110 en Proceedings of the Section on Survey Research Methods, American Statistical Association.

Groves, R. M. y Mick P. Couper 1993. "Unit Nonresponse in Demographic Surveys." Pp. 239-619 en Proceedings of the Bureau of the Census 1993 Annual Research Conference. Washington: Bureau of the Census.

Groves, R. M. y Mick P. Couper 1998. Nonresponse in Household Interview Surveys. Nueva York: Wiley.

Groves, R. M., Don A. Dillman, Jon L. Eltinge y J. A. Roderick Little. 2002. Survey Nonresponse. Nueva York: Wiley.

Groves, R. M., E. Singer y A. Corning 2000. "Leverage-Salience theory of survey participation." Public Opinion Quarterly 64:299-308.

Groves, R. M. y E. Peytcheva. 2008. "The impact of nonresponse rates on nonresponse bias." Public Opinion Quarterly 72:167-189.

Hox, J. J. y E. Deleeuw. 1994. "A Comparison of Nonresponse in Mail, Telephone, and Face-to-face Surveys." Quality and Quantity 28:329-344.

Instituto Nacional de Estadística. 2003. Evaluación de la falta de respuesta en la Encuesta de empleo del tiempo (TIC-H-2010). Madrid: INE. Consultado 16 de junio de 2011 (http://www.ine.es/daco/daco42/ empleo/evalfr.pdf).

Instituto Nacional de Estadística. 2009. Evaluación de la falta de respuesta en la Encuesta Europea de Salud 2009 (TIC-H-2010). Madrid: INE. Consultado 16 de junio de 2011 (http://www.ine.es/daco/daco42/ techog/evalfr10.pdf).

Instituto Nacional de Estadística. 2010a. Evaluación de la falta de respuesta en la Encuesta sobre equipamiento y uso de tecnologías de la información y comunicación en los hogares, 2010 (TIC-H-2010). Madrid: INE. Consultado 16 de junio de 2011 (http://www.ine.es/daco/daco42/techog/evalfr10.pdf).

Instituto Nacional de Estadística. 2010b. Evaluación de la calidad de los datos de la Encuesta de Población Activa 2010. Madrid: INE. Consultado 16 de junio de 2011 (http://www.ine.es/docutrab/eval_epa/evaluacion_epa10.pdf).

Instituto Nacional de Estadística. 2010c. Encuesta de Tecnologías de la Información en los Hogares. Madrid: INE. www.ine.es.

Laurie, H., R. Smith y L. Scott. 1997. "Strategies to Reducing Non-Response in a Longitudinal Panel Survey." Working Papers on the ESCR Research Centre on Micro-Social Change. Paper 97-12. University of Essex. Colchester.

Lin, I. y N. Cate Schaeffer. 1995. "Using Survey Participants to Estimate the Impact of Nonparticipation." Public Opinion Quarterly 59:236-258. 
Losilla, J. 2005. "Recogida de datos de una encuesta continua dirigida a los hogares: la EPA." en Trabajos de Campo en las encuestas del INE. Curso impartido en la Escuela de Estadística de las Administraciones públicas del Instituto Nacional de Estadística. 21-23 de junio.

Lynn, P. 1996. "Weighting for Non-Response." Pp.205-214 en Survey and Statistical Computing 1996, editado por R. Banks. Association for Survey Computing: Londres.

Lynn, Peter et al. 2002. "The effect of extended interview efforts on nonresponse bias." Pp. 135-148, en Survey Nonresponse, editado por Robert M. Groves et al. Nueva York: Wiley.

Kiecolt, K. J. y E. L. Nathan 1985. Secondary analysis of survey data. Thousand Oaks, CA: Sage Publications.

Kish, L. 1965. Survey Sampling. Nueva York: Wiley. Versión española en 1965: Muestreo de encuestas. México: Trillas.

Matsuo, H., J. Billiet, F. Loosveldt, F. Berglund y $\varnothing$ Kleven. 2010. "Measurement and adjustment of nonresponse bias based on non-response surveys: the case of Belgium and Norway in the European Social Survey Round 3." Survey Research Methods 4:121-126.

Metroscopia 2007. Informe técnico de la tercera ola en España (End of fieldwork report Spain. European Social Survey, 3rd round). Consultado 22 de julio de 2011 (http://www.upf.edu/ess/tercera-ed/).

Metroscopia 2009. Informe técnico de la cuarta ola en España (Final field report of the 4th round of ESS). Consultado el 22 de julio de 2011 (http://www.upf.edu/ess/_pdf/4a-ola/trabajo_campo/ESS08_FinalFieldReport.pdf).

Ministerio de Sanidad y Consumo 2006. Encuesta Nacional de Salud 2006. Metodología detallada. Madrid: Ministerio de Sanidad y Consumo.

Morton-Williams, J. 1993. Interviewer Approaches. Vermont: Darmouth.

National Centre for Social Research. 1999. How to Improve Survey Response Rates. A guide for Interviewers on the Doorstep (video) y How to Improve Survey Response Rates. A guide for Interviewers on the Doorstep (guía de utilización del vídeo). Londres: Sage.

Núñez Villuendas, A. 2005. "Incidencias de la entrevista personal en la investigación por encuesta." Revista Española de Investigaciones Sociológicas 109:219-236.

Peytchev, A., R. K. Baxter y L. R. Carley-Baxter. 2009. "Not All Survey Effort is Equal: Reduction of Nonresponse Bias and Nonresponse Error." Public Opinion Quarterly 73:785-806.

Riva, C., M. Torcal y L. Morales 2010. "Estrategias para aumentar la tasa de respuesta y los resultados de la Encuesta Social Europea en España." Revista Internacional de Sociología 68:603-635.

Rossi, P. H., James W. Wright y A. B. Anderson. 1983. "Sample surveys: History, current practice, and future prospects." Pp. 1-20 en Handbook of survey research, editado por Peter H. Rossi, James D. Wright, y Andy B. Anderson. San Diego: Academic Press.

Sánchez Carrión, J. J. 2000. La bondad de la encuesta: el caso de la no respuesta. Madrid: Alianza.

Schmeets, H. 2010. "Increasing Response Rates and the Consequences in the Dutch Parliamentary Election Study 2006." Field Methods 22:397-412.

Singleton, R. y B. Stratis 2005. Approaches to social research. Nueva York: Oxford University Press.

Skaland, B. 2011. "An alternative to the response rate for measuring a survey's realization of the tarjet population." Public Opinion Quarterly 75:89-98. 
Smith, T. W. 1981. The Hidden 25 percent: An Analysis of Nonresponse of the 1980 General Social Survey. GSS Technical Report 25. Chicago: National Opinion Research Center.

Smith, T. W. 1995. "Trends in Nonresponse Rates." International Journal of Public Opinion Research 7:157-171.

Smith, T. W. 2007. "Survey non-response in cross-national perspective: the 2005 ISSP non response survey." Survey Research Methods 1:45-54.

Statistics Sweden 1999. Journal of Official Statistics. Special issue on Survey Nonresponse 2.

Statistics Sweden 2001. Journal of Official Statistics. Special issue on Survey Nonresponse 2.

Steeh, C. G. 1981. "Trends in Nonresponse Rates: 1952-1979." Public Opinion Quarterly 45:40-57.

Sturgis, P. y P. Campanelli .1997. "Interviewer Intuition?: The Ability of Interviewers to Predict the Likelihood of Achieving an Interview before making Contact with Address Residents." Survey Methods Centre Newsletter 17: 3-6.

Stoop, I. A. 2005. The Hunt for the Last Respondent. Nonresponse in Sample Surveys. The Hague: Social and Cultural Planning Office of the Netherlands.

Stoop, I. Kele; J. Billiet, A. Koch y R. Fitzgerald. 2010. Improving Survey Response: Lessons Learned from the European Social Survey. Nueva York: Wiley.

Torcal, M., L. Morales y C. Riva. 2006. "Supervisión y control de calidad del trabajo de campo de la Encuesta Social Europea en España: Evaluación y resultados." Metodología de Encuestas 7:75-97.

Van den Brakel, J. A., R. Vis-Visschers y J. J. S. Schmeets. 2006. "An experiment with data collection modes and incentives in the Dutch Family and Fertility Survey for Young Moroccans and Turks." Field Methods 18:321-334.

Vehovar, V. 1999. "Field substitution and unit nonresponse." Journal of Official Statistics 15:335-350.

Willimack, Diane K., Howard Shuman, Beth-Ellen Pennell y James M. Lepkwoksi. 1995. "Effects of a prepaid nonmonetary incentive on response rates and response quality in a face-to-face survey." Public Opinion Quarterly 59:78-92.

Vidal Díaz de RAda es Licenciado y Doctor en Sociología, Licenciado en Investigación y Técnicas de Mercado, y profesor de Sociología en la Universidad Pública de Navarra, Departamento de Sociología. Es autor de diversos trabajos sobre metodología, sociología del consumo y comportamiento del consumidor; destacando entre sus últimas publicaciones "Manual del trabajo de Campo en la Encuesta" (2005); "Algunos problemas de la encuesta telefónica para la proyección electoral" (2007), "Estudio de las incidencias en la investigación mediante encuesta: el caso de los barómetros del CIS" (Con Núñez Villendas, 2007) y "Comparación entre los resultados obtenidos por encuestas personales y telefónicas: el caso de una encuesta electoral" (2010).

\section{RECIBIDO:07/02/2012}

ACEPTADO:08/07/2012

Publicado on-line: 11/03/2013 


\section{ANEXo \\ Definición de las incidencias en la entrevista en las encuestas del Centro de Investigaciones Sociológicas ${ }^{11}$}

1.2. Dificultad de acceso al edificio, casa, urbanización, etc. Contactos fallidos porque el portero, vecinos o presidente de comunidad de un inmueble impiden la entrada al edificio, o porque el guarda de seguridad de una urbanización supone una barrera de acceso infranqueable.

I.3. Viviendas en las que no hay nadie. Viviendas en las que no se puede contactar con persona alguna debido a que no responden a la llamada del entrevistador.

I.4. Viviendas en las que se niegan a recibir ninguna explicación. Hogares en los que se niegan a recibir explicaciones sobre el motivo de la presencia del entrevistador. La persona que abre la puerta se niega a recibir explicaciones sobre el motivo de la visita. También número de personas que rechazan hablar con el entrevistador después de haber llamado al portero automático.

1.5-6. Negativas de varones a realizar la entrevista. Tras contactar con el hombre o la mujer que cumple las características de sexo y edad, se niegan a ser entrevistados.

La negativa se refiere a la persona que cumple las características. Si cualquier otro miembro del hogar se niega a recibir explicaciones, es una incidencia que se anota en I.4

I.7. Contactos fallidos por no cumplir cuotas. No se llega a realizar la entrevista porque la persona con la que se contacta y accede a realizar la entrevista no cumple las condiciones para ser elegida (sexo 0 edad); 0 bien, porque aun existiendo esa persona en el hogar seleccionado no se encuentra en su domicilio.

I.8. Contactos fallidos por no ser una vivienda (oficinas, consultas médicas, etc.).

1.9. Viviendas de inmigrantes. Si el entrevistador, en el barrido de la sección, se encuentra con hogares de extranjeros sin derecho a voto deberá anotarlo como incidencia y no realizar la entrevista. Se trata de un contacto fallido porque los miembros de la vivienda no forman parte de la población objeto de estudio. Comienza a utilizarse a partir del barómetro de abril de 2001.

\footnotetext{
${ }^{11}$ Reproducido de Núñez Villuendas 2005:222-223.
} 